PERSPECTIVE

\title{
Why Residencies Should Fly: Towards a Logical Approach to Duty Hour Reform
}

\author{
Gina Luciano, MD, FACP' , Lydia Hambour, BTech, BBus², Paul Luciano, MBA ${ }^{3}$, \\ Eric Holmboe, MD', Sudeep Aulakh, MD ${ }^{7}$, Simon Fleming, MRCS, M.Sc ${ }^{5}$, and \\ Michael Rosenblum, $M D^{6}$
}

${ }^{1}$ Baystate HealthSpringfield, MA, USA; ${ }^{2}$ Rocket LabAuckland, New Zealand; ${ }^{3}$ JetBlue Airways CorporationNew York City, NY, USA; ${ }^{4}$ Accreditation Council for Graduate Medical EducationChicago, IL, USA; ${ }^{5}$ Barts and The London School of Medicine and DentistryLondon, UK; ${ }^{6}$ Ross University School of MedicineBridgetown, Barbados.

Since 2011, aviation has revolutionized their approach to safety. The aviation industry has adopted a multi-faceted approach to improve safety through decreasing duty hour limits and implementing processes to mitigate fatiguerelated errors as well as creating cultural shifts in responsibility for safety. These changes have been guided by data generated by quality-improvement methodology. In contrast, duty hour limits in graduate medical education have not yet seen dramatic data-driven reform. Key advancements in aviation fatigue mitigation and implications for residency education are explored in this article. Scientifically based processes to optimize duty hours, quality-improvement strategies to iteratively monitor and reform duty limits, systematic change focusing on a just culture, and financial disincentives and incentives as a catalyst for change are discussed.

KEY WORDS: graduate medical education; duty hours; fatigue; wellness; patient safety; aviation.

J Gen Intern Med 35(11):3333-7

DOI: $10.1007 / \mathrm{s} 11606-020-05894-\mathrm{z}$

(c) Society of General Internal Medicine 2020

\section{INTRODUCTION}

"You don't need kindergarten to know that a resident working a 36-hour shift is in no condition to make any kind of judgment call - forget about life-and-death." Sidney Zion ${ }^{1}$

Sidney Zion-father, lawyer, and New York Times writer-penned this statement in a 1989 op-ed, 5 years after his daughter, Libby Zion, died in New York Hospital. Mr. Zion, fervent to change the system that he felt was responsible for his daughter's death, convinced the New York County

Previous Presentations A pre-conference workshop describing elements of this manuscript was presented at the International Conference on Residencu Education in Halifax. Nova Scotia on October 17.2018.

Received November 8, 2019

Revised March 12, 2020

Accepted April 30, 2020

Published online May 29, 2020
District Attorney to investigate. A grand jury found fault with graduate medical training, and excessive resident work hours were considered to be a leading factor in Ms. Zion's demise. ${ }^{2}$

Public outcry after her death ignited debate about the relationship between resident fatigue, supervision, and patient safety and marked the start of duty hour reform. Since then, legislation in New York state, followed by the Accreditation Council for Graduate Medical Education (ACGME), has imposed duty hour changes. However, substantial debate remains about optimal duty hours and their impact on patient safety, resident wellness, and education.

Recently, studies such as FIRST and iCompare have examined duty hours. ${ }^{3,4}$ Balancing patient safety through enhanced continuity of care while optimizing resident wellness and education is a central theme. Each study examined the effect of standard duty hours (adherence to all ACGME requirements) versus flexible hours (extended shift duration nested within adherence to the 80 -h week, one day off in 7 , and call no greater than every third day). The FIRST trial, conducted in surgical residencies, found no differences in patient outcomes or resident well-being. ${ }^{3}$ Initial results from iCompare, evaluating internal medicine residents, demonstrated no significant differences between how residents spent clinical and educational time. ${ }^{4}$ However, interns in the flexible arm were more dissatisfied with their educational experience. Alarmingly, about $75 \%$ of interns in each arm reported moderate to high burnout levels.

While both studies are important steps towards delineating optimal duty hours, neither questions whether the 80 -h week in itself is problematic, with iCompare highlighting the staggering level of burnout. Excessive work hours and fatigue have been demonstrated to contribute to medical errors, ${ }^{5-7}$ injury among healthcare workers, ${ }^{8}$ and performance. ${ }^{9}$ FIRST and iCompare demonstrate that permutations of an 80-h work week yield similar results; however, would a study evaluating a 48-h week (European Working Time Directive regulating maximum hours worked) versus an 80-h week reveal different outcomes? More importantly, rather than seeking to randomize hours worked, can we determine the optimal number of hours required for safe care and effective education while ensuring physician well-being? Equally important, how can 
we transition from seeing time as an intervention (i.e., "a pill") to seeing hours as a resource?

Efforts to improve patient safety have applied innovations from aviation including standardized checklists, debriefings, and crisis management techniques. ${ }^{10-12}$ Although difficult to ascertain with great certainty, an estimated 44,000 to 251,454 deaths occur in US hospitals per year due to medical error. ${ }^{13-}$ ${ }^{15}$ In contrast, there has been only 1 death related to a United States passenger-carrying, scheduled airline since 2009, compared with 512 in the 10 years prior (not including fatalities resulting from acts of terrorism). ${ }^{16}$ Continued focus on safety improvements has led to a reduction in fatal airline accidents each decade since the beginning of the jet age, with fatigue mitigation in the last decade being a major aim. This is a noteworthy statistic given the steady growth in airline flights over the same time period. ${ }^{17}$ The marked disparity in safety between both industries led to an inquiry into how aviation has managed fatigue. Over the past decade, aviation has undergone a remarkable transformation in processes for mitigating fatigue to improve safety.

The logical question that follows is what can graduate medical education learn from aviation's recent revolution in fatigue management? While healthcare is markedly different than the aviation industry, some lessons may be adapted and modified to meet the needs of a complex healthcare environment. To better understand, we reached out to aviation experts (authors LH, who has international expertise in fatigue risk management as it relates to commercial airlines, and PL, who has financial expertise surrounding the impact of fatigue risk mitigation measures) as well as the chief pilot and a senior captain at JetBlue Airways Corporation headquarters to explore how lessons learned from aviation might apply to medical education to advance patient safety and physician wellbeing. We discuss advancements in aviation fatigue mitigation and implications for residency education.

\section{THE 80-H WORK WEEK}

"The specific ' 80 -hour week' was actually determined by a colleague on my porch and was based on the following informal reasoning: (1) there are 168 hours in a week; (2) it is reasonable for residents to work a 10-hour day for 5 days a week; (3) it is humane for people to work every fourth night; (4) subtracting the 50-hour week ( 10 hours per day $\times 5$ days) from 168 hours leaves 118 hours; (5) divide 118 by 4 (every fourth night) and add to the 50 hours and, eureka, that equals an 80 -hour week." Bertrand Bell ${ }^{18}$

On February 12, 2009, Colgan Air 3407 crashed killing all 49 passengers and crewmembers. With findings from the National Transport Safety Board (NTSB) that the pilots' performance was likely impaired because of fatigue and with subsequent public outcry, this incident called into question the importance of fatigue mitigation and prevention regulations. ${ }^{19}$ In response, in 2011, the Federal Aviation Administration (FAA) announced a new regulation, 14 CFR part 117, the first major modification of pilot rest, and duty limits in 50 years. $^{20,21}$ The new rule was formed utilizing an Aviation Rulemaking Committee (ARC) made up of representatives from industry, labor groups, and fatigue scientists. The FAA tasked ARC with providing recommendations that incorporated fatigue science, considered international standards, and introduced a new systems approach to fatigue risk management. The new rule outlined mandatory duty free periods: 10 -h rest periods before flight duty and $30 \mathrm{~h}$ of consecutive hours free on a weekly basis. ${ }^{22}$ Weekly, monthly, and annual limits on flight duties were established to mitigate effects of cumulative sleep deprivation. Additionally, time of day and jetlag were considered to incorporate the impact of circadian rhythms. Flightcrew would be required to declare fitness for duty prior to flying and airlines would develop and monitor fatigue education and fatigue reporting processes through an ongoing Fatigue Risk Management System (FRMS). Similar scientifically based fatigue regulations have also been introduced for commercial airlines in Europe, operating under the European Aviation Safety Authority, Australia, and South Africa.

Duty hour regulations in medicine originated from a collegial conversation on a porch. As a result of the Libby Zion case, New York State's Bell Commission determined that residents should not work more than $80 \mathrm{~h}$ or more than 24 consecutive hours. This became the standard in 2003 when the ACGME proposed the same 80-h limit as well as a regulation determining that residents could not work more than 24 consecutive hours with 6 additional hours to handoff care or attend educational conferences. Another regulation followed in 2011 limiting residents to $24+4$-h shifts ( $24 \mathrm{~h}$ of duty followed by up to $4 \mathrm{~h}$ to finish or transfer care or attend educational conferences) and interns to 16 -h shifts. ${ }^{23}$ In 2017, 16-h limits for interns were retracted and replaced with the $24+4$ regulation. ${ }^{24}$ As noted above, the FIRST and iCompare trials have shown that working beyond $80 \mathrm{~h}$ is associated with adverse events, leading the ACGME to reinforce the 80-h limit as mandatory for all residency and fellowship programs. ${ }^{25}$ However, while resident education on fatigue management is required, fatigue mitigation programs are not.

Duty hour requirements and mandatory rest periods are nonexistent for practicing physicians. Task complexity and time of day are rarely considerations, and physicians do not need to report fitness for duty prior to a shift. Systematic changes, based on science and data, are critical to the sustainability of our profession. 


\section{FATIGUE MITIGATION IS A SYSTEMS ISSUE}

"Failure saves lives. In the airline industry, every time a plane crashes the probability of the next crash is lowered by that. The Titanic saved lives because we're building bigger and bigger ships. So these people died, but we have effectively improved the safety of the system, and nothing failed in vain." Nassim Nicholas Taleb

Fatigue risk management in the airline industry is a systemlevel, iterative, quality-improvement process. It is a datadriven method to continuously monitor and manage fatiguerelated safety risks.

Multi-source data is collected from predictive modeling, pilot-generated reports from fatigue events, biometric monitoring, and aggregated safety events. Predictive modeling enables airlines to view each pilot's flight schedule to determine whether there will be adequate opportunity for sleep between flights. Modeling considers time of day, time zone, rest location, nap opportunities, and even commute times.

Schedules are proactively monitored so that the scientifically based limits are not exceeded even when unexpected delays occur. Schedules are planned below maximum limits to provide buffers. When delays are likely to exceed limits, "reserve" pilots are on-call to meet schedule demands.

Despite careful planning, fatigue risk can creep into the system. In the event that a pilot is unable to attain sufficient rest, $\mathrm{s} /$ he is required to call out and report. Each report generates a root cause analysis (RCA) to determine if a systems issue is to blame. Systems issues, such as a loud hotel or a continuously delayed flight, are analyzed to mitigate similar future issues.

Whereas predictive modeling addresses implications of scheduling, biometrics capture individualized data for actual sleep and wake times. Periodically, collected data is analyzed to identify major root factors contributing to fatigue. Root causes are addressed to further improve the working environment.

Resident schedules are generally designed in advance with little flexibility. If a resident is scheduled for an 80-h work week, the system has been designed with inherent duty hour violations. McCormick et al. have used aviation predictive modeling to determine resident effectiveness based on orthopedic residency schedules. They determined that implementing fatigue countermeasures has the capacity to improve resident effectiveness and that using fatigue optimization tools can provide data to better understand optimal schedules and potentially mitigate medical errors. ${ }^{26}$ However, most healthcare systems are not equipped to take into account real-time events that can delay a resident from leaving the hospital. Reconsidering fatigue management and hours as a quality-improvement process could help to redefine scheduling and its effects on training and safety.

\section{TOWARDS A “JUST" CULTURE}

"We tell physicians to get more sleep, eat more granola, do yoga, and take better care of yourself. These efforts are well intentioned. The message to physicians, however, is that you are the problem, and you need to toughen up." Tait Shanafelt ${ }^{27}$

Fundamental to FAR 117 is a systems approach whereby the airline is responsible for maintaining an environment to prevent and mitigate fatigue while crewmembers are expected to capitalize on that system. A culture has been built that promotes safe psychological and legal space for an individual to speak up, support, and grow a safe environment. Crewmembers are encouraged to report fatigue hazards and remove themselves from flying if fatigue could impact safety. This process is non-punitive and encouraged to support proactive identification and mitigation of fatigue-related hazards, and aims to identify risks before major incidents occur.

In healthcare, a common approach has been to "fix" the individual rather than build a model that prevents and mitigates fatigue-related risk. Time away from work and sleep have been related with improved resident well-being. ${ }^{28}$ While most strategies in medical education have promoted wellness curricula that often focus on the individual, fewer consider major infrastructural changes to create an environment that minimizes systematic reasons for fatigue. The Clinical Learning Environment Review 2018 national report found many training institutions continue to struggle to "identify and eliminate organizational factors that contribute to burnout."29

An area that requires systematic transformation is the mindset that fatigue and illness are "weaknesses." In a study investigating why clinicians work while sick, over $90 \%$ of respondents stated that they did not want to let their colleagues down and worried about whether there would be enough staff for patient care. Almost two-thirds of respondents feared ostracism. ${ }^{30}$ Most institutions have not built a system with reserve capacity for inevitable absences. In contrast, pilots are encouraged and expected to call out when sick or fatigued. A reserve system of on-call pilots (who are not otherwise working) exists to take the place of any pilot who is unable to legally fly. Medicine needs to create a similar reserve, so when a physician calls out it does not create chaos.

Innovative healthcare system redesign that thoughtfully considers how to borrow and adapt strategies that the airline aviation has implemented may be fundamental to mitigating fatigue and enhancing provider wellness and patient safety. For example, analyzing unintended consequences of our 
training model, such as the effect of long shifts or frequent educational transitions (i.e., rotations), can aid in transformative efforts. ${ }^{31}$ Rather than treating the symptom (provider), we need to focus our energies and expertise on treating the disease (system).

\section{FINANCIAL CONSIDERATIONS}

In aviation, the financial implications of breaking rules along with brand-damaging publicity are considered intolerable. Whenever a flight duty period rule is broken by any pilot, the airline is required to disclose this to the regulator, with a RCA and actions to ensure this incident does not recur. Repeated breaches risk major financial penalties. While airlines are individually responsible for designing, implementing, and refining their fatigue risk management programs, the FAA is responsible for overseeing each program and renewing the airline's Fatigue Risk Management Plan every 2 years. Failures to comply can result in the loss of an airline's operating certificate, meaning that every flight is grounded until the issue is resolved.

In contrast, residency programs have substantial leniency with interpreting duty hours. Residents can work up to $80 \mathrm{~h}$ on average and must have 1 day off in 7 on average. ${ }^{24}$ With many residents fearing that reporting duty hours accurately may self-sabotage their ability to graduate from an ACGMEaccredited program, capturing data is often challenging. ${ }^{32} \mathrm{~A}$ better understanding of how to reliably obtain this data within a non-punitive learning environment will be a necessary challenge to promoting and better understanding fatigue mitigation within healthcare.

Duty hour regulations and penalties in the aviation industry are mandated and monitored by the FAA. In medicine, the effect of monetary penalties could have a profound negative impact on residency programs and their sponsoring institutions but could catalyze change. In contrast, a proactive approach to mitigating fatigue could avoid programmatic and institutional hardship. Incentives to study, create, and monitor a fatigue mitigation system would be advantageous.

\section{CONCLUSION}

"Faced with the choice between changing one's mind and proving that there is no need to do so, almost everyone gets busy on the proof." John Kenneth Galbraith

The Colgan crash of 2009 marked the beginning of major change for US airline industries. With approximately 44,000 to 251,000 deaths attributed to medical error annually and suicidal ideation among physicians almost double that of the general population, it is time for a change. ${ }^{13,14,33}$ The impact of fatigue on residents and patients has an extraordinarily high cost for our healthcare system. Healthcare systems appear to be in disequilibrium as they compete over limited resources and have seemingly lost their connection to our most vital resources, our caregivers, and the teams that they work with to care for those in need.

Although optimal duty hours in medicine and their impact on fatigue and patient safety are not fully understood, investments in thoughtful change to support well-being and patient safety are needed. There are several lessons that key stakeholders, including the ACGME, AAMC, medical centers, insurers, regulators, and educators, can learn from aviation: (1) delineating optimal duty hours through evidence-based processes; (2) implementing a data-driven process to continuously monitor duty hours and its effects; (3) developing a system that critically evaluates the learning and working environment; and (4) re-examining financial disincentives and incentives surrounding duty hours and how these can be leveraged. Adapting these lessons to fit the complex nature of residency education and within the context of healthcare overall will require significant research, investment, and culture change. While obvious differences exist between flying a plane and providing patient care, medicine can and should learn from aviation and other fields working to improve working conditions, mitigate fatigue, and improve safety.

Corresponding Author: Gina Luciano, MD, FACP; Baystate Health Springfield, MA, USA (e-mail: Gina.Luciano@baystatehealth.org).

\section{Compliance with Ethical Standards:}

Conflict of Interest: None of the authors have any conflicts of interest pertaining to this work. In addition, none of the authors have any other conflicts of interest or disclosures except for Dr. Holmboe. Dr. Holmboe would like to disclose that he receives royalties from Elsevier for a textbook on assessment and is employed by the ACGME, an accreditation organization for graduate medical education.

\section{REFERENCES}

1. Zion S. Doctors know best? The New York Times. 1989. p.25

2. Asch DA, Parker RM. The Libby Zion case. One step forward or two steps backward? N Engl J Med. 1988;318(12):771-775.

3. Bilimoria KY, Chung Jw, Hedges LV, et al. National cluster-randomized trial of duty-hour flexibility in surgical training. $N$ Engl $J$ Med. 2016;374(8):713-727.

4. Desai SV, Asch DA, Bellini LM, et al. Education outcomes in a duty-hour flexibility trial in internal medicine. N Engl J Med. 2018;378(16):14941508.

5. Landrigan CP, Rothschild JM, Cronin JW, et al. Effect of reducing interns' work hours on serious medical errors in intensive care units. $N$ Engl J Med. 2004;351(18):1838-1848.

6. Barger LK, Ayas NT, Cade BE, et al. Impact of extended-duration shifts on medical errors, adverse events, and attentional failures. PLoS Med. 2006;3(12):e487.

7. West CP, Tan AD, Habermann TM, Sloan JA, Shanafelt TD. Association of resident fatigue and distress with perceived medical errors. JAMA. 2009;302(12): 1294-1300.

8. Lockley SW, Barger LK, Ayas NT, et al. Effects of health care provider work hours and sleep deprivation on safety and performance. Jt Comm J Qual Patient Saf. 2007;33(11 Suppl):7-18. 
9. Veasey S, Rosen R, Barzansky B, Rosen I, Owens J. Sleep loss and fatigue in residency training: a reappraisal. JAMA. 2002;288(9):11161124.

10. Gaba DM, Howard SK, Fish KJ, Smith BE, Sowb YA. Simulation-based training in anesthesia crisis resource management (ACRM): a decade of experience. Simulation \& Gaming. 2001;32(2):175-193.

11. Gawande A. The Checklist Manifesto: How to Get Things Right. New York: Metropolitan Books; 2010.

12. Nance JJ. Why hospitals should fly: the ultimate flight plan to patient safety and quality care. Bozeman, MT: Second River Healthcare Press; 2008.

13. Kohn LT, Corrigan J, Donaldson MS. To err is human: building a safer health system. Vol 6: National Academy Press Washington, DC; 2000.

14. Makary MA, Daniel M. Medical error-the third leading cause of death in the US. BMJ: British Medical Journal (Online). 2016;353.

15. Shojania KG, Dixon-Woods M. Estimating deaths due to medical error: the ongoing controversy and why it matters. BMJ Quality \& Safety. 2017;26(5):423-428.

16. National Transportation Safety Board. Aviation Statistics. https://www. ntsb.gov/investigations/data/Pages/aviation_stats.aspx . Accessed 15 feb 2020.

17. Boeing. Statistical Summary of Commercial Jet Airplane Accidents, Worldwide Operations 1959-2018. Technical report, Boeing. https:// www.boeing.com/resources/boeingdotcom/company/about_bca/pdf/ statsum.pdf. Accessed Feb 15, 2020.

18. Bell BM. Resident duty hour reform and mortality in hospitalized patients. JAMA. 2007;298(24):2865-2866.

19. National Transportation Safety Board. 2010. Loss of Control on Approach, Colgan Air, Inc., Operating as Continental Connection Flight 3407, Bombardier DHC-8-400, N200WQ, Clarence Center, New York, February 12, 2009 . NTSB/AAR-10/01. Washington, DC.

20. Barnett J. FAA announces pilot fatigue rule. https://www.cnn.com/ 2011/12/21/travel/pilot-fatigue-rule/index.html . Published 2011 Accessed 30 Jan 2018.

21. Trejos N. New pilot fatigue rules go into effect this weekend. https:// www.usatoday.com/story/todayinthesky/2014/01/03/pilot-fatiguemandatory-rest-new-faa-rules/4304417/. Published January 3, 2014. Accessed Jan 30, 2018.

22. Flightcrew member duty and rest requirements. In: Administration FA, ed. Vol 14 CFR Parts 177, 119, and 121. Federal Register: National Archives and Records Administration; 2012:330-403.
23. Rosenbaum L, Lamas D. Residents' duty hours-toward an empirical narrative. N Engl J Med. 2012;367(21):2044-2049.

24. ACGME. ACGME Common Program Requirements. https://www.acgme. org/Portals/0/PFAssets/ProgramRequirements/CPRs_2017-07-01.pdf. Published 2017. Accessed May 23, 2018

25. Nasca TJ. A Message from the ACGME President and CEO. https:// acgme.org/Newsroom/Newsroom-Details/ArticleID/9754/A-Messagefrom-ACGME-President-and-CEO-Dr-Thomas-J-Nasca November 19, 2019. Accessed Mar 2, 2020.

26. McCormick F, Kadzielski J, Evans BT, Landrigan CP, Herndon J, Rubash H. Fatigue optimization scheduling in graduate medical education: reducing fatigue and improving patient safety. J Grad Med Educ. 2013;5(1):107-111.

27. NEJM Catalyst. Physician Burnout: Stop Blaming the Individual. https://catalyst.nejm.org/videos/physician-burnout-stop-blaming-theindividual/ . Published 2016. Accessed 23 May 2018.

28. Raj KS. Well-Being in residency: a systematic review. J Grad Med Educ 2016;8(5):674-684.

29. Co JPT, Weiss KB, Koh NJ, Wagner R; CLER Program. CLER National Report of Findings 2018: Executive Summary. Chicago IL. Accreditation Council for Graduate Medical Education; 2018.

30. Szymczak JE, Smathers S, Hoegg C, Klieger S, Coffin SE, Sammons JS. Reasons why physicians and advanced practice clinicians work while sick: a mixed-methods analysis. JAMA Pediatr. 2015;169(9):815-821.

31. Bernabeo EC, Holtman MC, Ginsburg S, Rosenbaum JR, Holmboe ES Lost in transition: the experience and impact of frequent changes in the inpatient learning environment. Acad Med. 2011;86(5):591-598.

32. Drolet BC, Schwede M, Bishop KD, Fischer SA. Compliance and falsification of duty hours: reports from residents and program directors. $J$ Grad Med Educ. 2013;5(3):368-373.

33. Shanafelt TD, Hasan O, Dyrbye LN, et al. Changes in burnout and satisfaction with work-life balance in physicians and the general US working population between 2011 and 2014. Mayo Clin Proc. 2015;90(12):1600-1613.

Publisher's Note: Springer Nature remains neutral with regard to jurisdictional claims in published maps and institutional affiliations. 